\title{
TEM Study of Nano-Crystalline Al-Ni-La Powders Obtained by Gas Atomization
}

\author{
K. H. Foo, W.-A. Chiou and E. J. Lavernia*
}

Dept. of Chemical Engineering and Materials Science, University of California, Irvine, CA 92697

*Now at: Dept. of Chemical Eng. and Materials Science, University of California, Davis, CA 95616

Gas atomization, a process of atomization of liquid metals using high velocity inert gas jets, has been widely used in producing amorphous alloys as well as materials with well-developed microstructures due to its controllable cooling rates. As Al-based amorphous alloy with high Al content (> 80 at.\%) often exhibits durable tensile strength and efficient ductility ${ }^{1,2}$, an attempt of producing nano-crystalline and/or amorphous Al-Ni-La powders was thus undertaken. While characterization of gas atomized materials is numerous ${ }^{3}$, few studies have been reported on the study of small particles $(<25 \mu \mathrm{m})$ produced by this method. This paper presents the TEM study of small Al-Ni-La particles obtained from the gas atomization experiments.

Pure $\mathrm{Al}, \mathrm{Ni}$ and La with atomic ratio of $85: 10: 5$ were induction heated at $1100{ }^{\circ} \mathrm{C}$ in an argon atmosphere. The melt was then atomized by a concentric jet of $\mathrm{He}$ at the pressure of 200Ibs in a confined nozzle atomizer. Powders of $<25 \mu \mathrm{m}$ were collected using a ASTM 500 mesh sieve, and X-rayed using the Siemen X-ray diffractometer. A small portion of the powders was sonicated in alcohol, and 2-3 small drops of suspension were pipetted onto a holey carbon grid for TEM examination. However, the majority of particles were not electron transparent due to the thickness of large particles. To examine the detailed microstructure of a statistical number of these individual particles and to avoid possible artifacts on chemical analysis due to ion milling, dispersed powders were embedded in the SPURR epoxy and ultramicrotomed with a diamond knife. Ultra-thin sections were collected on holey carbon grids and examined in a TEM. For comparison, a portion of powders was embedded in EPO-TEK epoxy following by grinding/polishing, dimpling, and ion milling ${ }^{4}$.

$\mathrm{X}$-ray diffraction of sieved powders $(<25 \mu \mathrm{m})$ showed a typical very fine-grained and/or amorphous pattern (Fig.1). SEM images depicted well-rounded particles without specific morphology (Fig.2). However, results obtained using TEM revealed otherwise. TEM images of ultramicrotomed thin sections illustrated that a large amount of atomized powders was much less than $25 \mu \mathrm{m}$ in diameter (Fig. 3). Particle sizes ranged from about 5 to $500 \mathrm{~nm}$ with a majority around $30-50 \mathrm{~nm}$, and a few of more than $1 \mu \mathrm{m}$ in diameter. The diffraction contrast and electron diffraction showed these powders were nanocrystalline. TEM images of ultra-thin sections also depicted detailed internal microstructure of atomized particles. A large amount of atomized particles was composed of a few nanoparticles of 10-30 nm in diameter (Fig. 4). Well-developed facets can be clearly seen. An oxide layer also appeared on many particles (arrow in Fig. 4). Lattice fringes of $0.24 \mathrm{~nm}$ and EDS analysis indicated these particles were Al whereas the composition of other nanocrystalline particles vary. In addition, an abundant of elongated particles with many defects was observed in both ultramicrotomed and ion milled samples (Figs. 5 and 6). The lack of amorphous materials probably resulted from the composition un-homogeneity as shown by EDS and/or underspeed cooling rate during atomization. Nevertheless, gas atomization method provides an alternative technique of producing nano-/sub-micron particles with nanocrystalline grains.

1. A. Inoue et. al., Japan J. Appl. Phys., 27 (1988) L280, L176, also Pro. Mat. Sci. 43 (1998) 365.

2. K. R. Cardoso, et al., Mat. Sci. 7, Eng. A315 (2001) 89.

3. A. Zambon et. al., Materials Sci. and Eng. A304-306 (2001) 452.

4. W. A. Chiou et al., EMSA Proceedings, 49 (1991) 1116.

5. This research was supported by ONR and the Materials Characterization Center $\left(\mathrm{MC}^{2}\right)$ at UCI. 

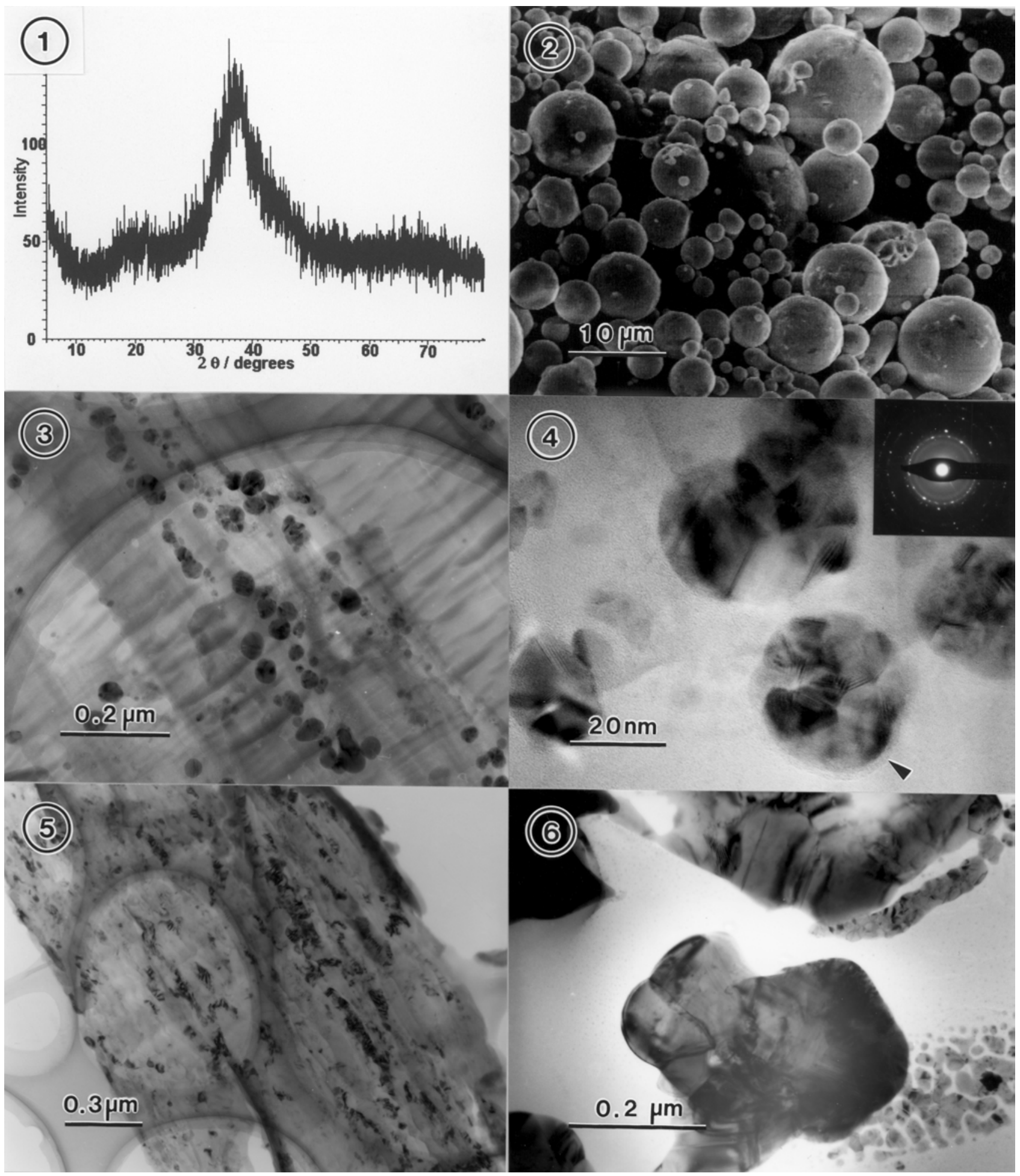

Fig. 1. X-ray diffractogram showing very fine-grained and/or amorphous-like pattern.

Fig. 2. SEM micrograph showing well-rounded shape of gas atomized particles.

Fig. 3. TEM micrograph revealing a large amount of very fine-grained particles.

Fig. 4. TEM micrograph depicting small particles consist of nano-grains with well defined facets.

Fig. 5. TEM micrograph of ultramicrotomed thin section revealing elongated particles.

Fig. 6. TEM micrograph of ion milled specimen showing small particles with many defects. 